University of Nebraska - Lincoln

DigitalCommons@University of Nebraska - Lincoln

\title{
$5-2011$
}

\section{The Effect of Axial Conduction on Heat Transfer in a Liquid Microchannel Flow}

Kevin D. Cole

University of Nebraska-Lincoln, kcole1@unl.edu

Barbaros Çetin

Middle East Technical University-Northern Cyprus Campus Kalkanlı, Turkey

Follow this and additional works at: https://digitalcommons.unl.edu/mechengfacpub

Part of the Mechanical Engineering Commons

Cole, Kevin D. and Çetin, Barbaros, "The Effect of Axial Conduction on Heat Transfer in a Liquid Microchannel Flow" (2011). Mechanical \& Materials Engineering Faculty Publications. 52.

https://digitalcommons.unl.edu/mechengfacpub/52

This Article is brought to you for free and open access by the Mechanical \& Materials Engineering, Department of at DigitalCommons@University of Nebraska - Lincoln. It has been accepted for inclusion in Mechanical \& Materials Engineering Faculty Publications by an authorized administrator of DigitalCommons@University of Nebraska Lincoln. 


\title{
The Effect of Axial Conduction on Heat Transfer in a Liquid Microchannel Flow
}

\author{
Kevin D. Cole \\ Mechanical Engineering Department, N104 Scott Engineering Center, University of Nebraska-Lincoln, \\ Lincoln, NE 68588-0656, USA (Corresponding author - tel 402 472-5857; email kcole1@unl.edu )

\section{Barbaros Çetin} \\ Mechanical Engineering, Middle East Technical University-Northern Cyprus Campus Kalkanl1, \\ Güzelyurt, TRNC Mersin 10, Turkey
}

\begin{abstract}
Analysis is presented for conjugate heat transfer in a parallel-plate microchannel. Axial conduction in the fluid and in the adjacent wall are included. The fluid is a constant property liquid with a fully-developed velocity distribution. The microchannel is heated by a uniform heat flux applied to the outside of the channel wall. The analytic solution is given in the form of integrals by the method of Green's functions. Quadrature is used to obtain numerical results for the local and average Nusselt number for various flow velocities, heating lengths, wall thicknesses, and wall conductivities. These results have application in the optimal design of small-scale heat transfer devices in areas such as biomedical devices, electronic cooling, and advanced fuel cells.
\end{abstract}

Keywords: wall conduction, microtube, microheat exchanger, laminar flow, conjugate heat transfer

\section{Introduction}

As fluid flow and heat transfer takes place at the microscale, many additional effects such as rarefaction, electro-viscous effects, viscous dissipation, and axial conduction need to be considered which can be neglected at the macro-scale. Rarefaction is important for small dimensions compared to the mean-free-path of the fluid (less than $5 \mu \mathrm{m}$ at atmospheric conditions), and is common for gas flows in microchannels. Electro-viscous effects are due to the interaction of the ions in the fluid with the electrical double layer (EDL) near the nonconducting channel wall [1], and is significant for liquid flow in microchannels with dimensions less than $5 \mu \mathrm{m}$ for deionized water. Viscous dissipation is the heating of the fluid due to the work done against the viscous forces. The effect of viscous dissipation can be important for flows with Reynolds number (Re) greater than 100 for microchannels [2].

From the heat transport point of view, the characteristic time for convection and conduction become comparable at the microscale, and the convection term no longer dominates the conduction term in the longitudinal direction. This is defined by flow for which the Peclet number (Pe) is not too large. Under this condition axial conduction in the fluid cannot be neglected as in the case of macrochannel flow. The effect of the axial conduction in the fluid becomes more pronounced as Pe decreases. The effect of axial conduction in the fluid on the heat transfer has been studied for both parallel-plate microchannel [3] and microtube [4,5] for boundary conditions defined by constant wall temperature $[3,4]$ and constant wall heat-flux $[3,5]$.

In conventional applications involving channels, the channel-wall thickness is very small compared to the hydraulic di- ameter of the channel; hence the heat transferred by conduction in the wall can be neglected compared to the convective heat transfer in many macroscale flows. However, in microchannels the thickness of the channel wall is usually equal in size or larger than the hydraulic diameter of the channel. Therefore the heat transferred in the wall by conduction cannot be neglected for the case of convective liquid flow in a microchannel, and the heat transfer mechanism becomes conjugate. The effect of axial conduction in the wall has been studied for macrochannel flows [6, 7]. In these studies, corresponding Pe values are high and as a consequence the axial conduction in the fluid was neglected. Maranzana et al. [8], Kroeker et al. [9], Li et al. [10], Kim and Kim [11, 12] studied the effect of axial conduction at the wall for the micro-channel heat sinks for both circular [9] and rectangular [8, 10-12] channel geometries. Maranzana et al. [8] studied the influence of axial conduction for parallel-plate geometry. Some of these studies $[8,11,12]$ had an assumption of constant convective heat transfer coefficient at the channel wall, that is, the linkage between the channel wall and the fluid flow was treated approximately. In contrast, Nonino et al. [13] analyzed the circular microtube using conjugate heat transfer, for which no approximation was introduced at the fluid-wall boundary. Recently, Kosar [14] analyzed the effect of the wall thickness and the wall material on heat transfer mechanism for a rectangular geometry with a fixed size, that is, only one geometry was studied. Although the results are presented in terms of nondimensional quantities, the analysis was dimensional. Moreover, the thermal boundary condition at the exit of the microchannel was specified convective flux, which was appropriate for the high Pe range and high Re range $(100<R e<1800)$ to which the work was restricted. 


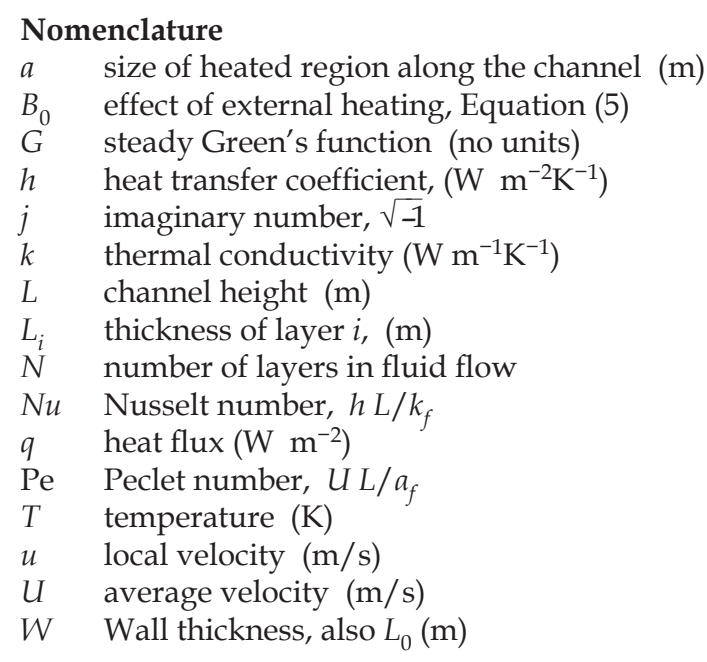

Greek

a thermal diffusivity $\left(\mathrm{m}^{2} \mathrm{~s}^{-1}\right)$

$\beta \quad$ wave number, Equation (8) $\left(\mathrm{m}^{-1}\right)$

$\delta \quad$ Dirac delta function

\section{Superscripts}

$+\quad$ dimensionless quantity

- $\quad$ spatial Fourier transform, Equation (9)

\begin{tabular}{ll}
\multicolumn{2}{l}{ Subscripts } \\
$f$ & fluid \\
$s$ & solid \\
$i$ & within layer $i$ \\
$w$ & wall value \\
$m$ & mean-flow value
\end{tabular}

A recent study by Perry et al. [15] involves a layered description of the fluid flow in a microchannel with wall effects. As this approach is close to that taken in the present work, a careful discussion of this paper is appropriate. Although Perry et al. [15] give results for several discrete fluid-flow values, temperature results are given for a fixed channel geometry with one heated-channel length, one wall thickness, and one wall conductivity. In contrast, in the present work comprehensive results are given for both temperature and Nusselt number for a variety of channel geometries and over a continuous range of fluid-flow values. As part of their solution, Perry et al. [15] use a finite-domain Fourier-transform which requires that they approximate the heated channel with a net-zero heat geometry. That is, the region of interest where heat is added is followed far downstream by a heat-out region, such that the net heat added is zero. Then they present temperature results for the heat-added region only. Unfortunately this approach may cause a distortion in the evolution of the temperature distribution along the channel at small Pe values, because of the far-reaching effects of axial conduction from the downstream heat-out region. In contrast, the infinite-domain Fourier transform used in the present work allow us to exactly characterize the heated channel and we present distortion-free temperature distributions, even at very small Pe values.

In this study, the convective heat transfer inside a parallelplate microchannel for low Pe number flow is analyzed. The fluid is assumed to be a constant-property liquid. The effect of the axial conduction both in the fluid and the wall is considered. Exact analytical solutions for the temperature distribution in the fluid and the wall are obtained by using Green's function method. The solution has the form of integrals and quadrature is used to obtain numerical values. Local and average values of the Nusselt number are determined for a range of fluid-flow values, for several heating lengths, for several wall thicknesses, and for several different wall materials. This information is expected to be useful in the analysis and design of micro-scale heat transfer devices.

The unique contributions of this paper are the following: fluid axial conduction is included for a low range of Pe $(0.1<P e<100)$ which has not been previously studied; natural inlet conditions are used (rather than specified temperature or flux); the analytic solution provides high precision, if desired; and, a wide range of results was explored because the quadrature could be evaluated rapidly compared to a fully-numeric solution.

\section{Temperature equations}

The equations describing the temperature in the parallelplate flow and in the adjacent wall are given in this section. The geometry is shown in Figure 1. The outside wall of the flow channel is heated, and the flow between parallel plates is fully-developed laminar. The plate spacing is $L$ and the wall thickness is $W$. The theoretical discussion given below is similar that for the steady-periodic theory developed previously [16], except here the frequency of heating is zero. The temperature satisfies the following equations:

$$
\begin{aligned}
& \frac{\partial^{2} T_{0}}{\partial x^{2}}+\frac{\partial^{2} T_{0}}{\partial y_{0}^{2}}=0 ; \quad 0<y_{0}<W ; \quad-\infty<x<\infty \\
& \frac{\partial^{2} T_{1}}{\partial x^{2}}+\frac{\partial^{2} T_{1}}{\partial y_{1}^{2}}=\frac{u\left(y_{1}\right)}{\alpha_{1}} \frac{\partial T_{1}}{\partial x} ; \quad 0<y_{1}<L / 2 ; \quad-\infty<x<\infty \\
& \left.k_{0} \frac{\partial T_{0}}{\partial y_{0}}\right|_{y_{0}=0}=p_{0}(x) ;\left.\quad \frac{\partial T_{1}}{\partial y_{1}}\right|_{y_{1}=L / 2}=0 \\
& T(x \rightarrow \pm \infty) \text { is bounded. }
\end{aligned}
$$

The specified heat flux, $p_{0}$, is applied at the outside of the wall at $y_{0}=0$. The centerline of the fluid flow is a zero-flux boundary to represent a channel that is heated symmetrically. The temperature far upstream and downstream $(x \rightarrow \pm \infty)$ will be bounded if function $p_{0}$ is non-zero over a finite region, which is the heating condition explored in this paper. If instead function $p_{0}$ is uniform over the half plane $(0<x<\infty)$, then the slope of the temperature $\partial T / \partial x$ will be bounded at $(x \rightarrow \infty)$.

The above differential equations for the temperature will next be recast as integral equations in each region (solid, fluid) with the method of Green's functions.

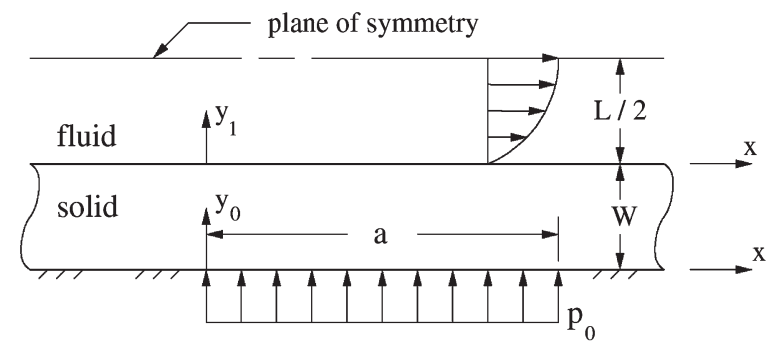

Figure 1. Geometry of parallel-plate microchannel flow heated through wall. 


\section{Green's function solution}

In this section the Green's function method will be used to seek the temperature distribution in the fluid and the adjacent wall. The Green's function in each region is a solution to the same equations and boundary conditions as those satisfied by the temperature, except that the boundary heating function $p_{0}(x)$ is replaced by a point heat source. The temperature solution is assembled by adding together many Green's functions in such a way that the heating distribution $p_{0}(x)$ is reconstructed from point sources. This adding together takes the form of a superposition integral, as shown below.

Let region 0 be a stationary solid heated at $y_{0}=0$ by a known heat flux $p_{0}(x)$ over region $(0<x<a)$. Let region 1 be a flowing fluid which is heated by contact with region 0 . Then the temperatures in each region may be formally stated with the method of GF in terms of known GF named $G_{1}$ and $G_{0}$ and unknown interface heat fluxes $q_{10}$ and $q_{01}$, as follows [17, chap. 3]:

$$
\begin{aligned}
& T_{1}\left(x, y_{1}\right)=\frac{1}{k_{1}} \int q_{01}\left(x^{\prime}\right) G_{1}\left(x-x^{\prime}, y_{1}, y_{1}^{\prime}=0\right) d x^{\prime} \\
& T_{0}\left(x, y_{0}\right)=\frac{1}{k_{0}} \int q_{01}\left(x^{\prime}\right) G_{0}\left(x-x^{\prime}, y_{0}, y_{0}^{\prime}=W\right) d x^{\prime}+B_{0}\left(y_{0}\right)
\end{aligned}
$$

where

$$
B_{0}\left(y_{0}\right)=\frac{1}{k_{0}} \int p_{0}\left(x^{\prime}, y_{0}^{\prime}\right) G_{0}\left(x-x^{\prime}, y_{0}, y_{0}^{\prime}=0\right) d x^{\prime}
$$

Here $B_{0}$ is the contribution to the temperature caused by the heat supplied on the outside of the wall, $p_{0}$. In the equations given above, the interface heat fluxes and the interface temperatures are unknown, but they are related by matching conditions at the interface between the regions. The heat flux entering region 1 leaves region 0 , and the temperatures match at the interface. That is,

$$
\begin{aligned}
& q_{01}(x)=-q_{10}(x) \\
& T_{1}(x, 0)=T_{0}(x, W)
\end{aligned}
$$

Next the Fourier transform will be used to strip away the integrals. The Fourier transform is defined by the following transform pair:

$$
\begin{aligned}
& \bar{T}(\beta)=\int_{-\infty}^{\infty} T(x) e^{-j \beta x} d x \\
& T(x)=\frac{1}{2 \pi} \int_{-\infty}^{\infty} \bar{T}(\beta) e^{j \beta x} d \beta
\end{aligned}
$$

Apply the Fourier transform to Equations (4)-(7) to obtain:

$$
\begin{aligned}
& \bar{T}_{1}\left(\beta, y_{1}\right)=\frac{1}{k_{1}} \bar{q}_{01}(\beta) \bar{G}_{1}\left(y_{1}, y_{1}^{\prime}=0\right) \\
& \bar{T}_{0}\left(\beta, y_{0}\right)=\frac{1}{k_{0}} \bar{q}_{01}(\beta) \bar{G}_{0}\left(y_{0}, y_{0}^{\prime}=W\right)+\frac{1}{k_{0}} \bar{p}_{0}(\beta) \bar{G}_{0}\left(y_{0}, y_{0}^{\prime}=0\right) \\
& \bar{q}_{01}(\beta)=-\bar{q}_{10}(\beta) \\
& \bar{T}_{1}(\beta, 0)=\bar{T}_{0}(\beta, W)
\end{aligned}
$$

If the GF are known in Fourier space, then an algebraic solution can be obtained for the unknown interface temperatures and heat fluxes. In the next section the GF for the fluid flow is found from a layered description of the fluid flow.

\subsection{Layered description of fluid flow}

It is possible to define one GF to describe the temperature in a channel with a continuously-varying velocity distribution. This approach requires use of a series involving the hypergeometric function with challenging series-convergence behavior [16]. In contrast, the layered approach given here involves a closed-form GF in each layer combined with a simple matrix solution. The continuous laminar flow will be replaced by a collection of flat layers, each one sliding over its neighbors with piecewise constant velocity. In each of these layers, application of the Fourier transform removes the x-coordinate, leaving heat conduction through layers along the y-direction. This method has been demonstrated with three layers in the fluid [15]. In the development below the fluid flow is described with an arbitrary number of layers with a method developed for heat conduction [17, chap. 9].

The layered geometry shown in Figure 2 has $N+1$ layers, numbered from 0 to $N$, with $N+1$ interfaces between the layers. If the zeroth layer is taken to be the wall with zero velocity, then the description of the wall can be included. Layers 1 through $\mathrm{N}$ are located in the laminar flow, with uniform velocity in each layer set to a value to produce a piecewise version of the laminar parabolic velocity distribution. Layer $i$ has thickness $L_{i}$ and thermal properties $k_{i}$ and $a_{i}$. Within layer $i$, the interfaces are at local coordinates $y_{i}=0$ and $y_{i}=L_{i}$. At the interfaces between the layers, let $q_{n m}$ represent the heat flux leaving layer $n$ and entering layer $m$. In the formulation given below, heating is caused by external heat at the outside of layer 0 and the dependence on Fourier parameter $\beta$ is dropped to streamline the development. Although in this formulation there is an insulated condition provided at the top of layer $\mathrm{N}$, another stationary wall could easily be added with heating or cooling added at that point.

Consider first the temperature in layer 0 evaluated at its interface with layer 1 :

$$
\bar{T}_{0}\left(L_{0}\right)=\frac{1}{k_{0}} \bar{G}_{0}\left(L_{0}, L_{0}\right) \bar{q}_{10}+\frac{1}{k_{0}} \bar{G}_{0}\left(L_{0}, 0\right) \bar{p}_{0}
$$

In layer $i ; i=1,2, \ldots N$ : the interface temperatures are:

$$
\begin{aligned}
& \bar{T}_{i}(0)=\frac{1}{k_{i}} \bar{G}_{i}(0,0) \bar{q}_{i-1, i}+\frac{1}{k_{i}} \bar{G}_{i}\left(0, L_{i}\right) \bar{q}_{i+1, i} \\
& \bar{T}_{i}\left(L_{i}\right)=\frac{1}{k_{i}} \bar{G}_{i}\left(L_{i}, 0\right) \bar{q}_{i-1, i}+\frac{1}{k_{i}} \bar{G}_{i}\left(L_{i}, L_{i}\right) \bar{q}_{i+1, i}
\end{aligned}
$$

In the last layer $(N)$ the temperature at the interfaces are

$$
\begin{aligned}
& \bar{T}_{N}(0)=\frac{1}{k_{N}} \bar{G}_{N}(0,0) \bar{q}_{N-1, N} \\
& \bar{T}_{N}\left(L_{N}\right)=\frac{1}{k_{N}} \bar{G}_{N}\left(L_{N}, 0\right) \bar{q}_{N-1, N}
\end{aligned}
$$

In the above expressions, symbol $p_{0}$ has been used for the external heat supplied that introduces heat into the microchannel. The Green's function $\bar{G}$. for the Fourier-space response of a layer with slug flow is given in the appendix.

In the above temperature expressions, all of the interface heat fluxes are initially unknown. The heat flux leaving one layer enters the adjacent layer, $\bar{q}_{i-1, i}=-\bar{q}_{i, i-1}$ and the temperature in adjacent layers is equal at each interface:

$$
\bar{T}_{i}(0)=\bar{T}_{i-1}\left(L_{i-1}\right) ; \quad i=1,2, \ldots, N
$$

Next Equations (14)-(18) are combined with Equation (19) to eliminate temperature. The result is a set of $N$ linear algebraic equations for the unknown heat fluxes, which may be stated in matrix form:

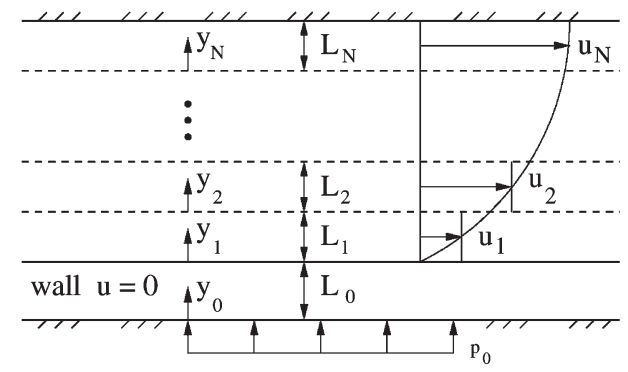

Figure 2. Geometry of slug-flow layers used to describe laminar flow in the microchannel. 


$$
\begin{gathered}
{\left[\begin{array}{ccccc}
C_{0}+C_{1} & -D_{1} & 0 & \cdots & 0 \\
-D_{1} & C_{1}+C_{2} & -D_{2} & \cdots & 0 \\
0 & -D_{2} & C_{2}+C_{3} & \ldots & 0 \\
\ldots & \ldots & \ldots & \ddots & -D_{N-1} \\
0 & 0 & \ldots & -D_{N-1} & C_{N-1}+C_{N}
\end{array}\right] \times\left[\begin{array}{c}
\bar{q}_{10} \\
\bar{q}_{21} \\
\bar{q}_{32} \\
\cdots \\
\bar{q}_{N, N-1}
\end{array}\right]} \\
=\left[\begin{array}{c}
-D_{0} \bar{p}_{0} \\
0 \\
0 \\
\cdots \\
0
\end{array}\right]
\end{gathered}
$$

Symbols $C_{i}$ and $D_{i}$ used in the above expression are given below:

$$
\begin{aligned}
& C_{i}=\frac{1}{k_{i}} \bar{G}_{i}(0,0)=\frac{1}{k_{i}} \bar{G}_{i}\left(L_{i}, L_{i}\right) \\
& D_{i}=\frac{1}{k_{i}} \bar{G}_{i}\left(0, L_{i}\right)=\frac{1}{k_{i}} \bar{G}_{i}\left(L_{i}, 0\right)
\end{aligned}
$$

For any multilayered system, it is now possible to calculate the $N$ unknown heat fluxes $\left(\bar{q}_{i j}\right)$ through all interfaces in the system. Cramer's rule may be used to solve for the $q$ 's for a laminar flow and adjacent wall approximated by two or three layers. However for four or more layers the well-known tridiagonal algorithm may be used. Once the heat fluxes are found, the temperature at any interface is given by Equations (14)-(18).

\subsection{Mean fluid temperature and nusselt number}

An important feature of the layered-fluid approach is that it allows for rapid computer computation of the mean temperature (bulk temperature) in the fluid. In an earlier study with a series solution for the fluid GF, evaluation of the mean temperature would have required additional evaluations of the series, at considerable computational cost.

The mean temperature is defined as a velocity-weighted average temperature in the fluid. For the parallel-plate channel the mean temperature is given in Fourier space by

$$
\bar{T}_{m}(\beta)=\frac{1}{U L} \int_{y=0}^{L} u(y) \bar{T}(\beta, y) d y
$$

where $u(y)$ is the local velocity and $U$ is the average velocity in the channel. For the layered description of the fluid used here, the single integral across the channel may be replaced by a series of integrals over each layer:

$$
\bar{T}_{m}(\beta)=\frac{1}{U L} \sum_{i=1}^{N} u_{i} \int_{y_{i}=0}^{L_{i}} \bar{T}_{i}\left(\beta, y_{i}\right) d y_{i}
$$

where $T_{i}$ is the temperature and $u_{i}$ is the uniform velocity in layer $i$. If the layers are sufficiently small, then the integral across each layer may be replaced, to good approximation, with the simple average of the temperatures at the two boundaries of each layer. That is,

$$
\bar{T}_{m}(\beta)=\frac{1}{U L} \sum_{i=1}^{N} u_{i} L_{i} \frac{1}{2}\left(\bar{T}_{i}(\beta, 0)+\bar{T}_{i}\left(\beta, L_{i}\right)\right)
$$

These layer-boundary temperatures are important because they may be computed at little cost from already-known quantities $\bar{q}_{m n^{\prime}} C_{i}$, and $D_{i}$.

The Nusselt number is the traditional way of presenting heat transfer results for channel flows. In the present work the Nusselt number and the local heat transfer coefficient are defined

$$
N u(x)=h(x) L / k ; \quad h(x)=\frac{p_{0}}{T_{w}(x)-T_{m}(x)}
$$

Here $p_{0}$ is the applied heat flux outside the wall, $T_{w}$ is the wall temperature at the fluid-solid interface, and $T_{m}$ is the fluid mean temperature defined above. When the wall is negligibly thin, then $p_{0}$ is the heat flux applied directly to the fluid.

The local heat transfer coefficient must be computed from the real-space temperature difference $\left[T_{w}(x)-T_{m}(x)\right]$, and the average heat transfer coefficient (or equivalently, the average Nusselt number) must be computed from the following integral:

$$
h_{a v}=\frac{1}{a} \int_{0}^{a} h(x) d x=\frac{1}{a} \int_{0}^{a} \frac{p_{0}}{T_{w}(x)-T_{m}(x)} d x
$$

It is important to note that because the Fourier transform is a linear operator applied to temperature, the Nusselt number cannot be computed in transform space and then inverse tranformed. This is equivalent to saying that the reciprocal of an integral is not the same as the integral of the reciprocal.

\section{Numerical considerations}

There were several numerical challenges in this project. As the Green's functions in Fourier space are complex valued functions, all the coding was carried out with variables of type double-precision complex in Fortran 77, compiled on a Sun UNIX workstation running the Solaris operating system.

Considerable programming effort was needed to obtain efficient evaluation of the Fourier-inversion integral in Equation (9), which is an improper integral (limits at infinity). Examination of the integrand indicated that it contains a sinusoidal factor, with zero crossings at $0, \pm \pi, \pm 2 \pi$, and so on. To take advantage of this form, the improper integral on $0<\beta<\infty$ was replaced by a summation of proper integrals, each of width $\pi$, beginning at $\beta=0$. Additional terms of this series were added until the fractional change in the magnitude of the running sum was less than a tolerance to provide 5-digit precision. The integral over $-\infty<\beta<0$ was handled in a similar way.

A numerical problem associated with steady convection heat transfer, that was not present in earlier work with steadyperiodic heat transfer, is that the Fourier-space GF for one layer has a singular point; specifically, $G \sim 1 / \beta$ near $\beta=0$ (refer to Equation (33) in the Appendix). A common method to deal with integrable singular points associated with a steady GF is to integrate analytically in the vicinity of the singularity [18]. Use of the analytic integral approach was not possible in the present work because of the numerical nature of the tridiagonal solution in Fourier space. Fortunately we found that by computing the difference between two temperatures in Fourier space, the near singular values in the vicinity of $\beta=0$ cancel out. With this understanding, it was possible to carry out the numerical integral for the Fourier inversion on subintervals $(-\pi, 0)$ and $(0, \pi)$ simply by using an open-ended Romberg algorithm, in which evaluation at the endpoints of the subinterval is avoided.

The precision associated with number of layers in the fluid was investigated by computing the wall temperature for a single specific geometry with 10, 20, 40, and 80 layers in the fluid.

Table 1. Change in wall temperature caused by the number of fluid layers, with $T^{+}=T_{\text {wall }} k_{f} /\left(p_{0} L\right)$ for geometry $P e=10, a / L=10, x / L=10$, $w=0$.

\begin{tabular}{lll}
\hline Layers & $T^{+}$ & \% Change \\
\hline 10 & 21.0686 & 0.231 \\
20 & 21.1057 & 0.055 \\
40 & 21.1150 & 0.011 \\
80 & 21.1173 & 0.000 \\
\hline
\end{tabular}




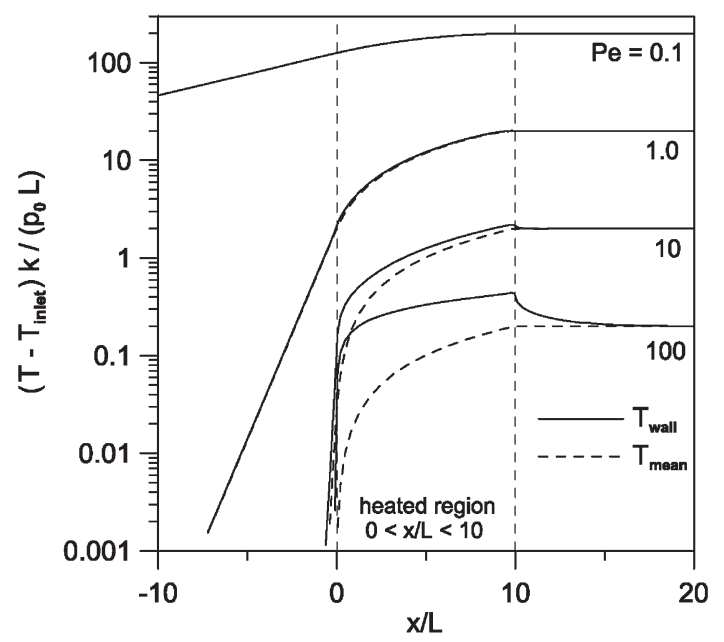

Figure 3. Normalized wall temperature and mean-fluid temperature versus position for fluid only (thin-wall case) and for heated region $(0<x / L<10)$.

The specific geometry is $P e=10, a / L=10, w=0$ (thin wall) and the wall temperature was evaluated at $x / L=10$. Refer to Table 1 . The percentage change in the wall temperature shows that ten fluid layers gives precision within $0.25 \%$ compared to the eighty-layer calculation. Based on this information ten fluid layers were used for the numerical results presented in this paper. Non-uniform spacing of the fluid layers was used, with thicker layers farther from the wall according to $L_{i} / L \approx i^{1.5}$ in order to equalize the velocity jump across successive layers. The results for a fixed number of fluid layers should be more precise at smaller Pe values for which the thermal boundary layer grows more quickly, spanning multiple fluid layers, therefore decreasing the impact of the layer thicknesses.

\section{Results and discussion}

\subsection{Fluid alone}

Numerical results are presented in this section for fluid flow alone, without an adjacent wall, in order to verify that the present method agrees with previous solutions, and to lay out some of the heat transfer phenomena that are important in the microchannel flow regime.

Figure 3 shows the wall temperature and the fluid mean temperature in the microchannel heated over region $(0<x / L<10)$ for several values of the Peclet number. Note that if the geometry of the channel is fixed, high Peclet number represents higher mean velocity for the same fluid; and if the mean velocity is fixed, higher Peclet represents a wider channel for the same fluid. At Pe $=0.1$ there is a large upstream region which is warmed by upstream axial conduction, and the temperature reaches its final value esssentially at the end of the channel. Axial conduction cannot be neglected at Pe small. However as Pe increases, convection becomes the dominant mechanism for transfer along the channel, visible in Figure 3 as less upstream heating (i.e. the upstream region does not feel the effect of the introduced heat).

In Figure 3 the maximum difference between the wall temperature and the mean temperature is small, about 0.25 dimensionless units, so that at $\mathrm{Pe}=0.1$ this difference is too small to be visible in the figure. As Pe rises however, the temperature values fall, and the small difference between the wall temperature and the mean temperature becomes visible on the semilog plot. At larger values of the Peclet number the local wall temperature on the heated region increasingly rises above the final downstream temperature. For larger Pe values the final

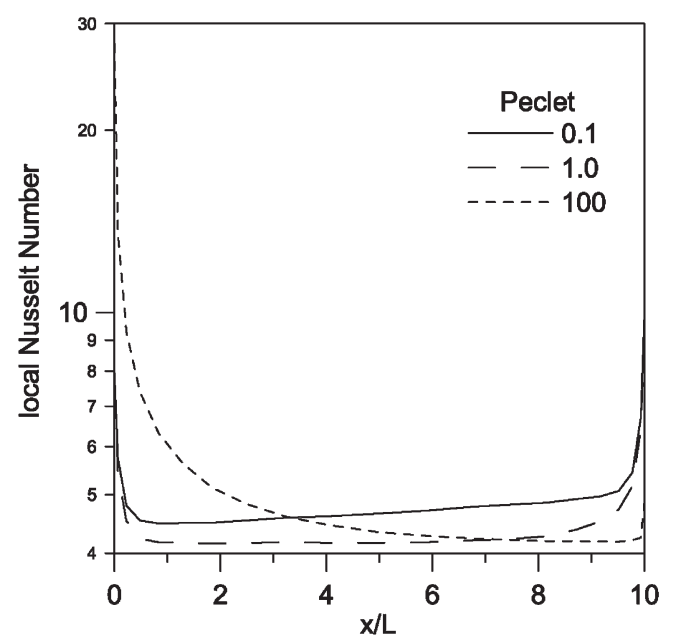

Figure 4. Local Nusselt number on the heated region $(0<x / L<10)$ for the fluid-only case showing the transition from convection-dominated behavior at $\mathrm{Pe}=100$ to axial-conduction dominated behavior at $\mathrm{Pe}=0.1$.

temperature is reached farther and farther downstream of the heated region. The wall temperature shown in Figure 3 agrees to four decimal places with an earlier publication by one of us on the fluid-only case [19].

It is important to note that the mean temperature distribution for each Peclet value shown in Figure 3 rises monotonically along the channel. The reason is that all the boundaries outside the heated region are insulated boundaries, so that heat added to the flow remains in the flow. As the Peclet number becomes smaller, representing smaller fluid mass flow, then the mean temperature reaches a higher final value in the channel. (If instead of the insulated boundaries given here, the far-field boundaries had been defined by a specified temperature, then for small Peclet number the steady temperature distribution would have been symmetric about the heated region, as heat conduction would have been dominant.)

The local Nusselt number is plotted in Figure 4 for fluid flow with a negligibly thin wall in a channel heated over region $0<x / L<10$. At $\mathrm{Pe}=100$ the Nusselt number is large at the entrance $(x=0)$ and falls toward the fully-developed value of 4.1175 [20, chap. 8]. It is important to note that reference [20] uses hydraulic diameter $2 L$ for defining the Nusselt number, while we use characteristic length $L$. The overall shape of the $\mathrm{Pe}=100$ curve agrees with macro-scale convection-dominated heat transfer. At $\mathrm{Pe}=1$, the Nusselt curve has a much smaller entrance region and quickly flattens out at about $x / L \approx 1$. But now there is a pronounced end-region uplift in the Nusselt values, caused by axial conduction in the fluid, whereby the temperature at the end of the heated region $(x / L<10)$ is affected by that in the unheated region $(x / L>10)$ (not shown). Finally, for $\mathrm{Pe}=0.1$, axial conduction is now a dominant factor, so much so that the rising Nusselt trend expected at the end of the heated region extends across the entire heated region, except for a very small entrance region. To repeat, $\mathrm{Nu}(\mathrm{x})$ rises along most of the channel flow when axial conduction dominates the heat transfer. The shape of the local $\mathrm{Nu}$ curve and the average $\mathrm{Nu}$ has a strong length dependence for low Pe flows.

The local Nusselt number is one way to view heat transfer behavior in the microchannel. Another view is provided by the normalized temperature difference between the wall and the mean flow, $\left[T_{w}(x)-T_{m}(x)\right] k /\left(p_{0} L\right)$. The normalized temperature difference is plotted in Figure 5 for the same conditions as Figure 4 , heating over $(0<x / L<10)$. The (normalized) temperature difference is valuable because unlike $\mathrm{Nu}(\mathrm{x})$, the temperature difference is well-defined outside of the heated region (it is equal to the reciprocal of the Nusselt number inside 


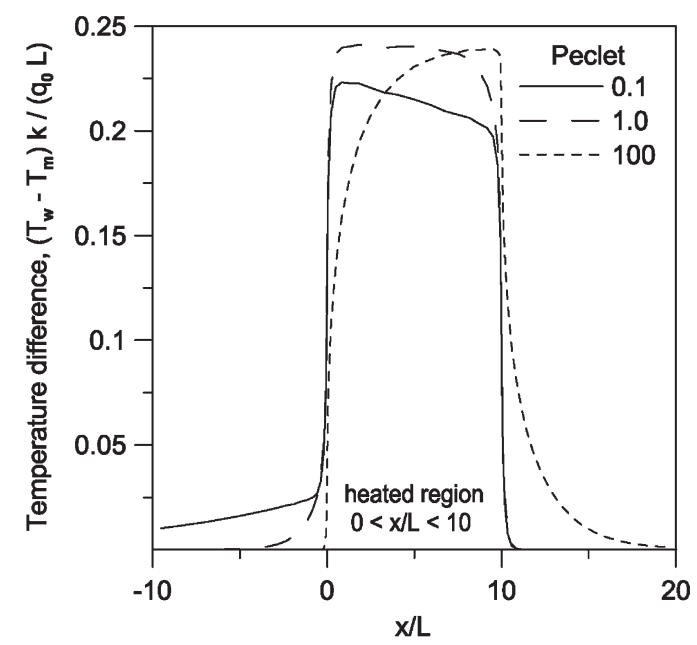

Figure 5. Normalized temperature difference between the wall and the mean flow, $\left[T_{w}-T_{m}\right] k /\left(p_{0} L\right)$, versus axial position for the thinwall case for three velocity (Peclet) values. On the heated region $(0<x / L<10)$ this temperature difference is the reciprocal of the local Nusselt number.

the heated region). The temperature difference curve for $P e=100$, which is convection dominated, shows zero temperature difference upstream of the heated region, a rising temperature difference on the heated region, and a slowly falling temperature difference in the downstream region. In the downstream region the fluid temperature across the channel slowly equilibrates through cross-stream conduction. In contrast, the $\mathrm{Pe}=0.1$ curve has a long upstream region where the fluid is heated by axial conduction upstream, a slowly falling temperature difference in the heated region, and a rapid drop to zero in the downstream region. The Pe $=1$ curve in Figure 5 shows intermediate behavior as expected. As the Peclet number decreases from 100 to 0.1 , the reversal of temperature-difference trends in all three regions (upstream, on the heater, downstream) shows how bulk convection and axial conduction have competing effects on heat transfer along the channel.

The temperature response upstream and downstream of the heated region are important to discuss because previous work in simulating microchannel flow has involved the specified-temperature entrance condition for the fluid (see for example [15]). A specified temperature at the fluid entrance is appropriate only for macro-channel flow, that is, when the boundary layer assumption is valid and fluid axial conduction may be neglected. However, when axial conduction is important (as shown above for $P e<100$ ), a specified entrance temperature will artificially lower the upstream temperature difference and raise the Nusselt number, compared to the natural entrance condition provided here. In a similar way, use of a specified temperature at the fluid exit will distort the predicted Nusselt number in this region.

\subsection{Fluid with wall present}

In this section the heat transfer is applied to the microchannel through a wall of thickness $W$. The effect of the wall is to provide another avenue for heat to travel axially along the channel. The amount of heat carried away from the heated region by the wall is controlled by the wall thickness and the wall thermal conductivity. In terms of overall trends on the Nusselt number, the presence of the wall is quite similar to the effects of axial conduction in the fluid discussed in the previous section.

Figure 6 shows the spatial average Nusselt number versus fluid flow (Pe) for four wall thicknesses and for $k_{\text {wall }} / k_{\text {fluid }}=2.5$. The thin-wall case is also shown for comparison. The Nusselt

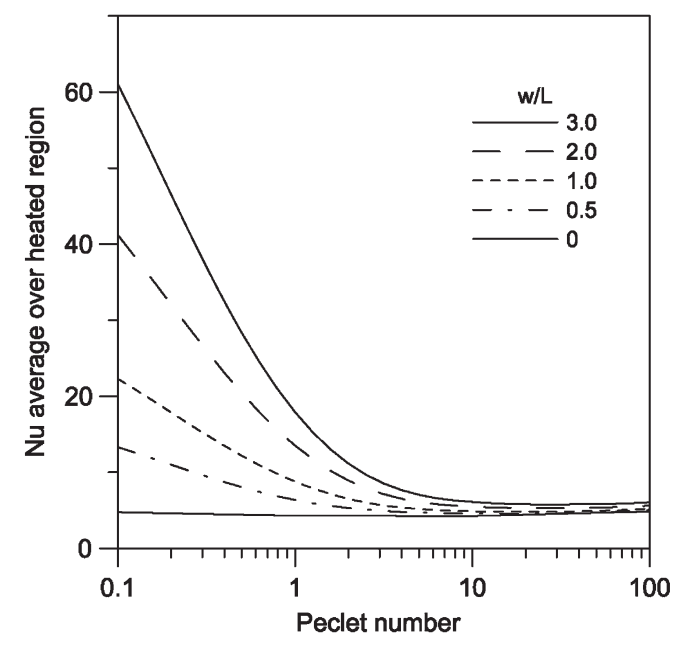

Figure 6. Average Nusselt number on the heated region $(0<x / L<10)$ for $k_{\text {wall }} / k_{\text {fluid }}=2.5$ for various wall thicknesses.

values are larger for small-Pe flows, and larger for the thickwall case. The addition of the wall causes heat to move axially upstream to pre-warm the flow. The effect is greatest at small Pe where the bulk fluid motion is weak. For $P e>10$, where bulk motion is stronger, a change in wall thickness produces a much smaller increase in the average Nusselt values. Similar curves for other $k_{\text {wall }} / k_{\text {fluid }}$ (not shown) were also investigated, which contain similar trends, except that the increase in $\mathrm{Nu}$ values at small $\mathrm{Pe}$ is magnified as $k_{\text {wall }} / k_{\text {fluid }}$ increases. The curves also show the expected trend that for high Pe flows the effect of the wall can be neglected, or conversely, the effect of the wall is strongest in water flow when the Pe is less than 10.

Figure 7 shows the effect of $k_{\text {wall }} / k_{\text {fluid }}$ on the average Nusselt number at wall thickness $W / L=1.0$ and for heating length $a / L=10$. The conductivity ratios are typical for conventional materials. That is, values $k_{\text {wall }} / k_{\text {fluid }}=2.5,25,250$ and 500 have been chosen to represent the cases in water flow where the channel wall is glass, stainless steel, silicon, and copper, respectively. The no-wall case (denoted $k_{\text {wall }}=0$ to represent $w=0$ ) is also shown for comparison. As Peclet number decreases the average $\mathrm{Nu}$ values increase due to axial conduction effects. There is a small uplift in average Nu values for $P e>10$ when $k_{\text {wall }} / k_{\text {fluid }}$ is small, which is caused by the competing effects of flow entrance and flow exit in this relatively short heat

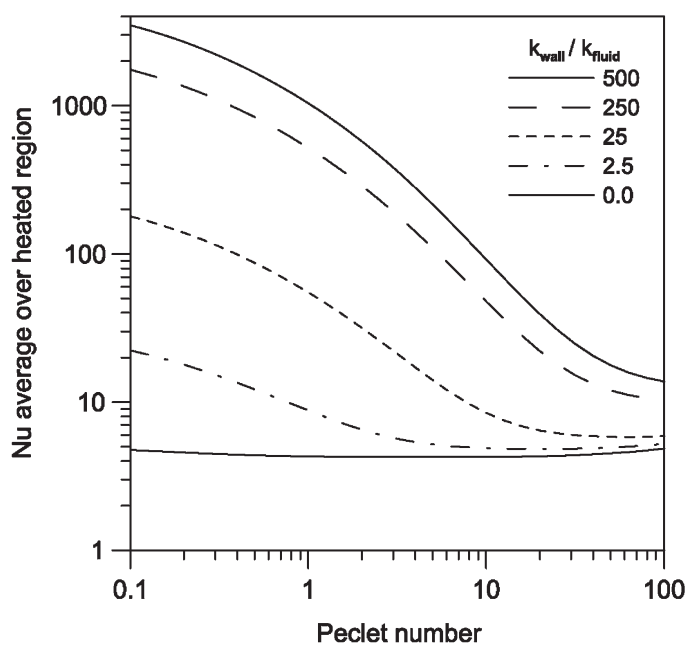

Figure 7. Average Nusselt number on the heated region $(0<x / L<10)$ for wall thickness $W / L=1.0$ showing the effect of various wall conductivity ratios $k_{\text {wall }} / k_{\text {fluid }}$. 


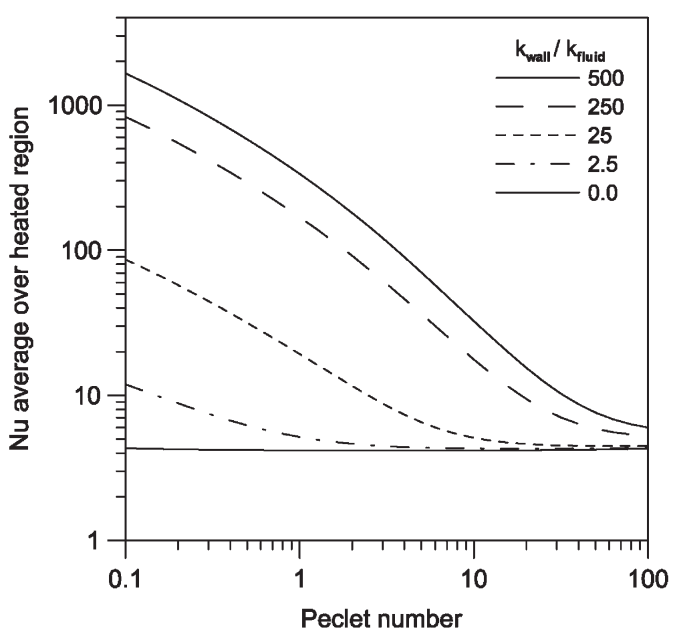

Figure 8. Average Nusselt number on the heated region $(0<x / L<50)$ for wall thickness $W / L=1.0$ for various wall conductivity ratios $k_{\text {wall }} / k_{\text {fluid }}$.

exchanger. Figure 8 shows the effect of $k_{\text {wall }} / k_{\text {fluid }}$ on the average Nusselt number for the same conditions as Figure 7 but for a longer heat exchanger with heating length $a / L=50$. For this curve the peak $\mathrm{Nu}$ values are lower and the no-wall curve has a less pronounced uplift at $P e>10$ because the entrance and exit effects are less evident in this longer heat exchanger. Figure 9 shows average Nusselt number versus Peclet number for the same conditions as [Figure 7, Figure 8] but now the heat exchanger is very long with $a / L=250$. The peak $\mathrm{Nu}$ values at small Peclet number are even lower, and the curves for the lower values of $k_{\text {wall }} / k_{\text {fluid }}$ coalesce into a single curve as Peclet number increases. This is because the primary effect of the wall is to add axial conduction at the entrance and exit, which now are a tiny fraction of the heat exchanger, except for the very highest $k_{\text {wall }} / k_{\text {fluid }}$ case. Note also in Figure 9 the nowall curve is essentially flat and at the fully-developed value, again because the entrance and exit effects are negligible for this case. These curves demonstrate that if the heating section of the microchannel is long relative to the height of the channel, and the if the flow is a high Pe flow (e.g. Pe $>100$ ), the effect of the wall conduction is not significant. However, if the heating section of the channel is short, or the flow is low Pe flow, the effect of the wall conduction cannot be neglected. This effect is more pronounced when the wall material is more conductive relative to the working fluid.

Although plots of results are important for visualizing trends, equipment designers and researchers find great value in precise numerical values. Table 2 provides numerical values of the spatial average Nusselt number for the heated flat-plate channel for several heated lengths, wall thicknesses, fluid-flow values, and conductivity ratios. The no-wall case has been included for comparison. Some of the larger $\mathrm{Nu}$ values in Table 2 have been rounded off to four significant digits in keeping with the demonstrated precision of the results.

\section{Conclusions}

In this study conjugate heat transfer for a flow of a constant-property liquid in a parallel-plate microchannel is analyzed including the axial conduction in the fluid and in the adjacent wall. The microchannel is heated by a uniform heat flux applied to the outside of the channel wall. The closed form solutions for the temperature field and the $\mathrm{Nu}$ are obtained in the form of integrals by the method of Green's functions. The effect of Pe, channel length, wall thicknesses, and wall conductivities on the local and average $\mathrm{Nu}$ are discussed.

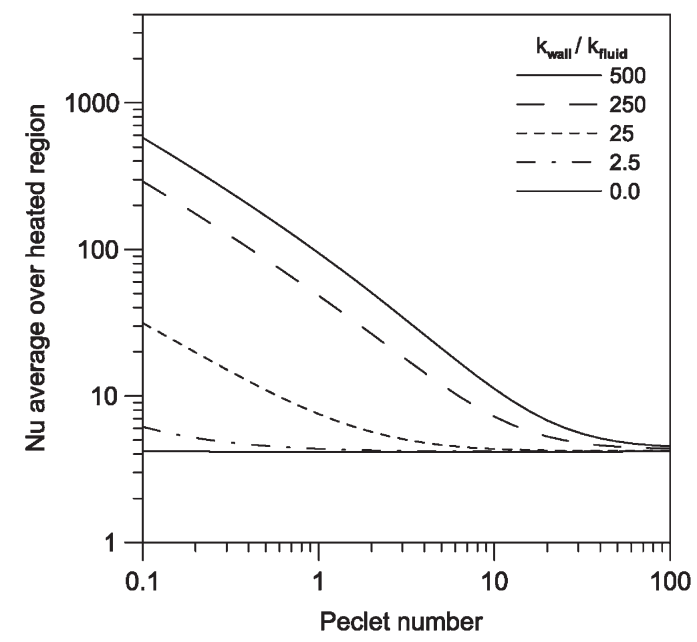

Figure 9. Average Nusselt number on the heated region $(0<x / L<250)$ for wall thickness $W / L=1.0$ for various wall conductivity ratios $k_{\text {wall }} / k_{\text {fluid }}$.

Table 2. Spatial average Nusselt number showing the effect of heated length $a / L$, wall thickness $w / L$, Peclet number, and conductivity ratio.

\begin{tabular}{|c|c|c|c|c|c|}
\hline$k_{w} / k_{f}$ & $\mathrm{Pe}$ & $w / L$ & $\begin{array}{r}a / L \\
10 \\
\end{array}$ & 50 & 250 \\
\hline \multirow[t]{4}{*}{ - } & 0.1 & 0 & 4.75 & 4.33 & 4.19 \\
\hline & 1 & 0 & 4.30 & 4.18 & 4.16 \\
\hline & 10 & 0 & 4.26 & 4.17 & 4.16 \\
\hline & 100 & 0 & 4.87 & 4.30 & 4.18 \\
\hline \multirow[t]{16}{*}{2.5} & 0.1 & 0.5 & 13.32 & 7.86 & 5.01 \\
\hline & & 1 & 22.32 & 11.91 & 6.15 \\
\hline & & 2 & 41.21 & 20.30 & 8.80 \\
\hline & & 3 & 61.09 & 28.85 & 11.62 \\
\hline & 1 & 0.5 & 6.37 & 4.61 & 4.24 \\
\hline & & 1 & 8.79 & 5.17 & 4.35 \\
\hline & & 2 & 13.53 & 6.44 & 4.61 \\
\hline & & 3 & 17.93 & 7.76 & 4.88 \\
\hline & 10 & 0.5 & 4.60 & 4.24 & 4.17 \\
\hline & & 1 & 4.89 & 4.30 & 4.19 \\
\hline & & 2 & 5.48 & 4.42 & 4.21 \\
\hline & & 3 & 6.10 & 4.54 & 4.23 \\
\hline & 100 & 0.5 & 5.06 & 4.34 & 4.19 \\
\hline & & 1 & 5.23 & 4.37 & 4.20 \\
\hline & & 2 & 5.61 & 4.45 & 4.21 \\
\hline & & 3 & 6.05 & 4.52 & 4.23 \\
\hline \multirow[t]{16}{*}{250} & 0.1 & 0.5 & 864.50 & 414.60 & 146.10 \\
\hline & & 1 & 1752.00 & 830.20 & 290.50 \\
\hline & & 2 & 3615.00 & 1674.00 & 582.00 \\
\hline & & 3 & 5578.00 & 2530.00 & 875.90 \\
\hline & 1 & 0.5 & 262.60 & 85.94 & 25.40 \\
\hline & & 1 & 521.30 & 169.20 & 48.36 \\
\hline & & 2 & 1018.00 & 331.60 & 93.74 \\
\hline & & 3 & 1479.00 & 485.80 & 137.70 \\
\hline & 10 & 0.5 & 25.98 & 10.41 & 5.46 \\
\hline & & 1 & 47.84 & 17.69 & 7.22 \\
\hline & & 2 & 90.81 & 32.27 & 11.13 \\
\hline & & 3 & 132.30 & 46.51 & 15.16 \\
\hline & 100 & 0.5 & 8.13 & 4.86 & 4.30 \\
\hline & & 1 & 10.31 & 5.25 & 4.38 \\
\hline & & 2 & 13.90 & 6.01 & 4.53 \\
\hline & & 3 & 17.12 & 6.76 & 4.69 \\
\hline
\end{tabular}


It was found that the effect of the axial conduction in the channel wall is important: (i) when the microchannel has a small length-over-height ratio; (ii) when the Pe is small; (iii) when the wall thickness relative to the channel height is large; and, (iv) when the wall conductivity of the wall material is high relative to the thermal conductivity of the working fluid. For high Pe flow (e.g. Pe > 100) together with a wall with lower thermal conductivity, the effect of the axial conduction in the wall is negligible. One application for these results is the optimal design of microchannel heat exchangers.

\section{Appendix A. Green's function}

The GF for the steady temperature in slug flow between parallel-plates satisfies the following differential equations (subscript $i$ has been suppressed to simplify the notation)

$$
\frac{\partial^{2} G}{\partial x^{2}}+\frac{\partial^{2} G}{\partial y^{2}}-\frac{u}{\alpha} \frac{\partial G}{\partial x}=-\delta\left(x-x^{\prime}\right) \delta\left(y-y^{\prime}\right) ; \quad 0<y<L
$$

Here $u$ is the spatially-uniform velocity in the $x$-direction. Unit-amplitude steady heating is introduced at point $\left(x^{\prime}, y^{\prime}\right)$ and the response is observed at point $(x, y)$. The boundary conditions are

$$
\begin{aligned}
& \text { At } x \rightarrow \pm \infty, \quad G \text { is bounded. } \\
& \text { At } y=0, \quad \frac{\partial G}{\partial y}=0 \\
& \text { At } y=L, \quad \frac{\partial G}{\partial y}=0
\end{aligned}
$$

This case Y22, which is to say both boundaries are of the second kind. For the present work, the spatial-Fourier transform of this GF is needed. First use a simple change of variable to replace $\left(x-x^{\prime}\right)$ by $x$. Then apply the Fourier-transform, Equation (13), to the above differential equation, to find

$$
\frac{d^{2} \bar{G}}{\partial y^{2}}-v^{2} \bar{G}=-\delta\left(y-y^{\prime}\right)
$$

where $v^{2}=\beta^{2}+j \beta u / a$. Note that although real-space $G$ is dimensionless, Fourier-space $\bar{G}$ has units of meters. The solution for the GF in Fourier space is given by (see [17], chap. 9, or [21])

$$
\bar{G}\left(y, y^{\prime}\right)=\frac{\left(e^{-v\left(2 L-\left|y-y^{\prime}\right|\right)}+e^{-v\left(2 L-y-y^{\prime}\right)}\right)}{2 v\left(1-e^{-2 v L}\right)}+\frac{\left(e^{-v\left(\left|y-y^{\prime}\right|\right)}+e^{-v\left(y+y^{\prime}\right)}\right)}{2 v\left(1-e^{-2 v L}\right)}
$$

Although this GF was defined for slug flow, the stationary wall is also described by this GF for the special case $u=0$ (zero velocity). To find the GF in $x$-space an inverse Fourier transform is needed. However, in the present work the GF is used to find the temperature in Fourier-transform space and then the inverse-Fourier transform is applied to the temperature solution.

\section{References}

[1] B. Cetin, B. E. Travis, and D. Li, Analysis of the electro-viscous effects on pressure-driven liquid flow in a two-section heterogeneous microchannel, Electrochimi. Acta 54 (2008), pp. 660-664.

[2] G. L. Morini, Scaling effects for liquid flows in microchannels, Heat Transfer Eng. 27 (4) (2006), pp. 64-73.

[3] H. E. Jeong and J. T. Jeong, Extended Graetz problem including streamwise conduction and viscous dissipation in microchannels, Int. J. Heat Mass Transfer 49 (2006), pp. 2151-2157.

[4] B. Cetin, A.G. Yazicioglu, and S. Kakac, Fluid flow in microtubes with axial conduction including rarefaction and viscous dissipation, Int. Comm. Heat Mass Transfer 35 (5) (2008), pp. 535-544.

[5] B. Cetin, A. G. Yazicioglu, and S. Kakac, Slip-flow heat transfer in microtubes with axial conduction and viscous dissipation An extended Graetz problem, Int. J. Thermal Sci. 48 (9) (2009), pp. 1673-1678.

[6] E. J. Davis and N. W. Gill, The effects of axial conduction in the wall on heat transfer with laminar flow, Int. J. Heat Mass Transfer 13 (1970), pp. 459-470.

[7] S. Mori, Y. Kawamura, and A. Tanimoto, Conjugated heat transfer to laminar flow with internal heat source in a parallel plate channel, Canadian J. Chem. Eng. 57 (1979), pp. 698-703.

[8] G. Maranzana, I. Perry, and D. Maillet, Mini- and micro-channels: influence of axial conduction in the walls, Int. J. Heat Mass Transfer 47 (2004), pp. 3993-4004.

[9] C. J. Kroeker, H. M. Soliman, and S. J. Ormiston, Three-dimensional thermal analysis of heat sinks with circular cooling micro-channels, Int. J. Heat Mass Transfer 47 (22) (2004), pp. 4733-4744.

[10] J. Li, G. P. Peterson, and P. Cheng, Three-dimensional analysis of heat transfer in a micro-heat sink with single phase flow, Int. J. Heat Mass Transfer 47 (2004), pp. 4215-4231.

[11] S. J. Kim and D. Kim, Forced convection in microstructures for electronic equipment cooling, J. Heat Transf. 121 (1999), pp. 639-645.

[12] D. K. Kim and S. J. Kim, Averaging approach for microchannel heat sinks subject to the uniform wall temperature condition, Int. J. Heat Mass Transfer 49 (2006), pp. 695-706.

[13] C. Nonino, S. Savino, S. Del Giudice, and L. Mansutti, Conjugate forced convection and heat conduction in circular microchannels, Int. J. Heat Fluid Flow 30 (2009), pp. 823-830.

[14] A. Kosar, Effect of substrate thickness and material on heat transfer in microchannel heat sinks, Int. J. Thermal Sci. 49 (2010), pp. 635-642.

[15] I. Perry, Y. Jannot, D. Maillet, and B. Fiers, Effect of velocity distribution on external wall temperature field for a flat microchannel, Exp. Heat Transfer 23 (2010), pp. 27-43.

[16] K. D. Cole, Steady-periodic heating in parallel-plate microchannel flow with participating walls, Int. J. Heat Mass Transfer 53 (2010), pp. 870-878.

[17] K. D. Cole, J. V. Beck, A. Haji-Sheikh, and B. Litkouhi, Heat Conduction Using Green's Functions (2nd ed.), Taylor and Francis, New York (2010).

[18] S. A. Harman, Conjugate heat transfer: effects of fluid axial conduction and the two-component wall, M.S. Thesis, University of Nebraska, Mechanical Engineering Dept., 1998.

[19] A. Haji-Sheikh, J. V. Beck, and K. D. Cole, Steady-state Green's function solution for moving media with axial conduction, Int. J. Heat Mass Transfer 53 (2010), pp. 2583-2592.

[20] W. M. Kays, M. E. Crawford and B. Weigand, Convective Heat and Mass Transfer (4th ed.), McGraw-Hill, New York (2005).

[21] K. D. Cole, Green's Function Library: http://www.greensfunction.unl.edu ; accessed August 23, 2010. 